\title{
Dinámica espacio-temporal del esfuerzo en una pesquería de buceo artesanal multiespecífica y sus efectos en la variabilidad de las capturas: Implicaciones para el manejo sostenible
}

\author{
Helven Naranjo Madrigal ${ }^{*}$ \& Silvia Salas Márquez² \\ 1. Programa de Doctorado en Ciencias Marinas. CINVESTAV del IPN. Unidad Mérida 97310, Apartado Postal 73 \\ Cordemex, Yucatán, México; helvenn@hotmail.com \\ 2. Centro de Investigación y de Estudios Avanzados del IPN, Km 6 antigua Carretera a Progreso, Apartado Postal 73 \\ Cordemex, Mérida 97310, Yucatán, México; ssalas@mda.cinvestav.mx \\ * Correspondencia
}

Recibido 26-II-2014. Corregido 07-V-2014. Aceptado 10-VI-2014.

\begin{abstract}
Spatio-temporal dynamics of fishing effort in a multi-species artisanal diving fishery and its effects on catch variability: Insights for sustainable management. Artisanal diving fisheries are a source of income, employment and food security of coastal areas in many countries. Understanding the dynamics of these fisheries, including the spatial and temporal dynamics of fishing effort, gears and species can help to address the challenges involved in fisheries management. We aimed to analyze the differences in fishing strategies undertaken by fishers that use two different diving methods (hookah and free diving), the conditions and their potential impacts on catches when adjustments to those strategies are applied over time. For this, detailed information of fishing operations from artisanal boats in the North Pacific coast of Costa Rica was analyzed in two fishing seasons (2007-2008 and 2011-2012). Data were collected by onboard observers (fishing site, fishing time, species composition, depth and visibility). Additionally, interviews with divers were applied to obtain information of price per species, species volume and fishing operations. From the total number of trips during both seasons, hookah diving was represented by a sample size of $69.3 \%$, while free diving, with a sample of $41.9 \%$. More than 15 species were identified in each fishing season. Nevertheless, three categories had substantial contributions in both seasons with differences in the proportions for each case: green lobster (Panulirus gracilis), octopus (Octopus sp.) and parrotfish (Scarus perrico and S. ghobban). It is worth noting that an important proportion of catch was retained by fishers for personal consumption purposes, including species of high commercial value. Additional night diving activity, increased the number of dives from one season to another. Besides, cooperation processes in free diving fishing operations, and changes in fishing effort between seasons, defined important changes in fishing strategies. Potential causes of changes in fishing strategies and the implications for management to ensure the sustainability of these fisheries in the long term are discussed. Rev. Biol. Trop. 62 (4): 1565 1586. Epub 2014 December 01.
\end{abstract}

Key words: Costa Rica, diving artisanal fishery, fishing strategies, fishing effort allocation, Generalized Additive Models, sustainable fisheries management.

La sostenibilidad a largo plazo de las pesquerías en el mundo depende de la capacidad de las naciones en controlar efectivamente el esfuerzo de pesca, regular los mercados dado un incremento en la demanda de productos, así como promover buena gobernanza (Worm et al., 2009, Alfaro-Shigueto et al., 2010). Sin embargo, en pesquerías artesanales de países subdesarrollados, la implementación efectiva de regulaciones y generación de estrategias de manejo es limitada, con poca inversión económica en investigación y ordenamiento, generando por lo tanto alta incertidumbre en la sostenibilidad de los recursos que sostienen 
estas pesquerías y consecuentemente el bienestar de quienes de ellas dependen (Andrew et al., 2007).

Diversos factores han incentivado un aumento en la presión de pesca, entre ellos el aumento en la demanda y precios de las especies de mayor valor comercial, regímenes de acceso abierto, ausencia de empleos alternativos en las zonas rurales y costeras, aunados al fácil acceso a las poblaciones de especies comerciales, dado el uso de diversos métodos de pesca (Caddy \& Defeo, 2003; Salas, Chuenpagdee, Seijo, \& Charles, 2011). En muchos casos los métodos de pesca empleados demandan baja inversión y costos operativos bajos, lo que aumenta las posibilidades de expandir áreas de operación (Caddy \& Defeo, 2003; Salas \& Gaertner, 2004; Salas, Chuenpagdee, Seijo, \& Charles, 2007). Estos métodos son accesibles a pobladores de comunidades rurales de bajos recursos que utilizan la pesca como estrategia de sobrevivencia, estilo de vida y seguridad alimentaria (Béné, 2003; Plagányi et al., 2013).

La dinámica de pesquerías artesanales multi-específicas, especialmente en zonas costeras tropicales, se caracterizan por una gran variación espacio-temporal de sus descargas, diversidad de artes y especies objetivo, alta dispersión de las actividades pesqueras a lo largo de la costa, y gran variabilidad en los rendimientos, lo que complica su evaluación y manejo (Salas et al., 2007; Salas et al., 2011). Por esta razón, en la evaluación de estas pesquerías es necesario incluir aspectos relativos a las operaciones de pesca asociadas al uso de artes de pesca mixtos, que permitan discernir cambios en la composición de las capturas, y en la dinámica espacio-temporal del esfuerzo de pesca (Salas \& Gaertner, 2004; Monroy, Salas, \& Bello-Pineda, 2010), así como conocer los patrones de respuesta del pescador a cambios en el ambiente o regulaciones, y su efecto en la variabilidad de las capturas (Moses, Udoidiong, \& Okon, 2002; Voges, Gordoa, \& Field, 2005; Katsanevakis, Maravelias, Vassilopoulou, \& Haralabous, 2010; Stevenson, Brian, \& Dierking, 2011; Horta e Costa et al., 2013).
En Costa Rica un $80 \%$ de la flota es artesanal, concentrada principalmente en aguas del Pacífico con alta incidencia en especies bento-demersales (Herrera-Ulloa, VillalobosChacón, Palacios-Villega, Viquez-Portuguéz, \& Oro-Marcos, 2011). La naturaleza dispersa y aislada de los puertos a lo largo de la costa limita conocer los procesos de operación de la flota artesanal. A la fecha, no existen estudios de dinámica del esfuerzo pesquero de las flotas que explotan recursos bento-demersales, que permitan conocer la composición de especies, y el comportamiento espacio-temporal de las embarcaciones, generando condiciones de acceso abierto en la pesca con los riesgos que esto involucra. Como en otras pesquerías artesanales en América Latina las pesquerías costarricenses han estado bajo condiciones de desatención por parte de las autoridades (Salas et al., 2011). Por ejemplo, en el Pacífico Norte, existen regulaciones que vedan la captura del pepino Isostichopus fuscus y caracol Strombus galeatus, y otras que restringen el esfuerzo y la captura de la langosta verde Panulirus gracilis, sin embargo, éstas no se cumplen (Naranjo, 2011; Naranjo, 2012).

En este contexto se exploran las estrategias de pesca de pescadores artesanales que capturan recursos bento-demersales de poca movilidad y alto precio en el mercado. Dadas estas características de las especies y los métodos de pesca empleados, existen los riesgos de colapso de pesquerías importantes como el pepino de mar y la langosta (Purcell, Mercier, Conand, Hamel, \& Toral-Granda, 2011; Eriksson, de la Torre-Castro, \& Olsson, 2012). El empleo de buceo como un método común para la captura de estas especies en amplias regiones de América Latina resulta relevante por sus implicaciones sobre el recurso y sus usuarios (Arceo \& Seijo, 1991; Béné \& Tewfik, 2001; FAO, 2006; Huchim, Fraga, \& Salas, 2012).

En este estudio se planteó conocer si existen diferencias en las estrategias de pesca desarrolladas por pescadores que emplean como método de pesca dos modalidades: hookah y buceo libre. También se busca conocer si se dan adaptaciones de estas estrategias en el 
tiempo en términos de composición de especies y esfuerzo de pesca ejercido. Para este fin, se contrastó la información de dos temporadas de pesca de los diferentes métodos de buceo, y se analizó la composición de especies, las fuentes de variación de las capturas, los patrones espacio-temporales del esfuerzo y se identificó las especies objetivo. Se partió del supuesto de que los pescadores desarrollan adaptaciones a sus estrategias en el tiempo y el espacio, cuando ven afectados sus retornos económicos con cambios en las capturas (Béné \& Tewfik, 2001; Daw, 2008). Se discuten los resultados de este trabajo con el fin de derivar aspectos relevantes en el manejo sostenible de especies susceptibles y de la actividad pesquera en general.

\section{MATERIALES Y MÉTODOS}

Área de estudio: El presente estudio se realizó en Playa Lagarto ubicada en la provincia de Guanacaste, Costa Rica $\left(10^{\circ} 07^{\prime} .23^{\prime \prime}\right.$ $\left.\mathrm{N}-85^{\circ} 47^{\prime} .97^{\prime \prime} \mathrm{W}\right)$ durante las temporadas de pesca del 2007-2008 y 2011-2012. En esta región operan embarcaciones de $12 \mathrm{~m}$ de eslora que emplean buceo con hookah $(\mathrm{BH})$ como método de pesca. También operan pescadores que emplean buceo libre (BL), desplazándose a las zonas de pesca directamente desde la costa. Los patrones específicos a las áreas que acceden estos pescadores no han sido reportados.

Fuentes de información: La información que se utilizó en este estudio se basó en la colecta de información fina de los viajes de pesca de pescadores durante sus jornadas de pesca. Esta involucró entrevistas y observación participativa en campo (zona de desembarque y a bordo de embarcaciones). También se emplearon estadísticas pesqueras de fuentes oficiales, así como de intermediarios y grupos de pescadores.

Se aplicó una entrevista general a los pescadores de 15 embarcaciones y a los buzos que operan con $\mathrm{BL}$ al momento del desembarque en las temporadas de pesca del 2007-2008 y 2011-2012. Se registró la captura con el fin de conocer su composición y se tomaron datos de precio por especie y costos de viaje. También se registró el número de viajes y tiempo de pesca según el método utilizado.

Se tomó un volumen de desembarques para el caso del BH equivalente a un $55.6 \%$ del total de viajes en la temporada 2007-2008, y un $81 \%$ en la temporada 2011-2012. En la temporada 2007-2008 la recolecta de datos comprendió 11 meses (noviembre-octubre), a excepción del mes de junio que no se realizaron viajes de pesca debido a condiciones ambientales adversas. La temporada 2011-2012 tuvo una duración de nueve meses (de noviembre a junio) debido a que las fuertes lluvias impidieron las actividades. Para el caso del BL, se obtuvo una muestra del $41.9 \%$ con respecto al total de viajes en las dos temporadas de pesca. En Playa Lagarto desembarcaron un promedio de 13 embarcaciones durante las dos temporadas de pesca y trabajaron un promedio de 27 buzos que emplean BL durante las dos temporadas de pesca analizadas.

En la temporada 2011-2012 algunas embarcaciones realizaron viajes de noche, estrategia no observada en la temporada previa. Estos viajes se categorizaron con el nombre de BHN (buceo con hookah de noche) y se realizaron un total de 17 viajes, de los cuales se obtuvo una muestra de 12 viajes (70.5\%). Sin embargo, solo se utilizó esta información para comparar la composición de especies en la captura con respecto a los demás métodos de buceo $(\mathrm{BH}$ y $\mathrm{BL}$ ), debido a que el bajo número viajes limitó el análisis de la asignación espacial y temporal del esfuerzo pesquero para el buceo nocturno.

Con el fin de caracterizar las operaciones de pesca durante los viajes se determinó la profundidad (m), el tiempo efectivo de pesca (horas), visibilidad horizontal en la columna de agua $(\mathrm{m})$ la cual se registró mediante un disco Secchi y cinta métrica, número de inmersiones por viaje para el caso del BH y las coordenadas de los sitios de pesca donde realizaron las inmersiones fueron registradas con un GPS $\left(\right.$ marca Garmin $\left.{ }^{\circledR}\right)$. Conversaciones con los pescadores ayudaron a complementar la información relativa a los sitios visitados durante los viajes de pesca, tiempo promedio de la faena y 
condiciones de visibilidad. La participación de observadores en algunas de las faenas de pesca como inmersiones y viajes de pesca proporcionó información detallada sobre factores que incidieron en el desarrollo de la faena de pesca en las escalas espacial y temporal.

\section{Composición de la captura y especies} objetivo: La composición de las capturas descargadas por los buzos fue definida por las categorías comerciales, como localmente se manejan. Se separaron las especies destinadas a la comercialización de las retenidas para consumo familiar o trueque (denominada a lo largo del trabajo como subsistencia).

El valor de la captura se estimó como el producto del precio pagado por el intermediario al pescador en la zona de desembarque (precio de playa) multiplicado por el volumen descargado de cada especie. Para asignar valor económico a la categoría de subsistencia que incluyó una mezcla de especies que se comercializan y otras no comercializadas se utilizó el precio promedio del total de las especies comercializadas. De esta manera se pudo obtener el valor de la captura que se retuvo para consumo personal.

Dados los cambios observados en las estrategias de los pescadores de una temporada de pesca a otra (tres años después), se analizó el reflejo de ese cambio en la composición de las capturas entre temporadas de pesca. Para este fin se utilizó el diseño multivariado propuesto por Warton, Wright, y Wang (2012) mediante el cual se ajustan Modelos Lineales Generalizados (MLGs) a cada una de las especies simultáneamente. Se usaron rutinas presentes en el paquete mvabund (Wang, Neuman, Wright, \& Warton, 2012) en el programa estadístico $R$ (2012) versión 2.15.0.

Se analizó si existía un efecto significativo del factor temporada sobre el promedio de captura en la matriz de especies (Ecuación 1). La distribución de la captura no mostró una distribución normal por lo que se usó una distribución Gamma (Maunder \& Punt, 2004) con función de enlace logarítmica para asegurar que los valores ajustados fueran positivos (Zuur, Leno, Walker, Saveliev, \& Smith, 2009).

Una vez obtenido el resultado del ajuste de los MLGs para cada especie de forma simultánea con respecto a la temporada, se implementó un procedimiento de remuestreo con el cual se obtuvieron aproximaciones validas de valores de $p$ lo que permitió probar la significancia estadística. El procedimiento de remuestreo permite generar una distribución de posibles salidas mediante el reordenamiento y muestreo de los datos (Manly, 1991; Edgington \& Ongheda, 2007). Se utilizó el método de remuestreo a los residuales, ya que permite disminuir en forma eficiente la varianza provocada por el proceso de remuestreo (Whitley, 1994). Este diseño multivariado propuesto tiene la ventaja de identificar patrones fuertes en los datos sin necesidad de realizar trasformaciones basadas en índices de distancia, además de tomar en cuenta la relación entre la varianza y la media de captura para cada especie, ya que la primera tiende a aumentar con respecto a la segunda (Warton et al., 2012).

$$
\log (\text { ucaptura })=\beta_{o}+\beta \text { temporada }+\varepsilon(\text { Ec. } 1)
$$

Las especies objetivo de las dos temporadas y para los dos métodos fueron definidas ajustando MLGs separados para la captura y el valor de captura de cada especie con respecto a los viajes de cada método (Ecuación 2). Luego se sumó el radio de probabilidad (RP) estadístico para cada caso. Se aplicó un procedimiento de remuestreo, similar al propuesto en el párrafo anterior, para evaluar la significancia de este RP multivariado. La suma del estadístico RP es análoga a la suma del $\mathrm{F}$ estadístico basado en análisis de varianza (Edgington \& Ongheda, 2007). Este procedimiento permitió estimar el efecto de la diferencia en el promedio de cada especie dentro de los viajes.

$$
\log (\mu \text { viajes })=\beta_{o}+\beta \text { captura }+\varepsilon(\text { Ec. } 2)
$$

Fuentes de variación de las capturas: En este estudio variables como la visibilidad en la columna de agua, ubicación geográfica, 
y formas de operacionales (número de inmersiones y el número de buzos por viaje) se consideraron como variables relevantes en la definición de la función de producción, además del tiempo de pesca. El tipo de muestreo empleado en este estudio, permitió obtener información a nivel de inmersión, lo que ayudó a evaluar las fuentes de variación en las capturas en el caso de pesquerías bento-demersales donde el buceo es el método principal de pesca.

Para conocer el efecto de distintas variables en la contribución a la variabilidad en la captura total por viaje de pesca, se construyeron funciones de producción por método de buceo. Se ajustaron Modelos Aditivos Generalizados (GAMs) mediante el paquete estadístico $m g c v$ en el programa estadístico $\mathrm{R}$, siguiendo procedimientos de pruebas, ajustes, y gráficos propuestos por Zuur et al. (2009). Para la selección del mejor modelo se evaluó la magnitud de la devianza explicada por las variables dentro de los modelos para cada método y la puntuación del procedimiento de validación cruzada generalizada (GCV), permitió comparar las variables con mejor desempeño (Wood, 2006). Mediante este procedimiento se pudieron definir las mejores unidades de esfuerzo que tuvieron mayor peso para cada método.

Se utilizó como variable respuesta el volumen total $(\mathrm{kg})$ de las especies capturadas por viaje (pulpo, langosta, ostión, pez loro, entre otras). Las variables predictivas seleccionadas con base en la observación participativa de los viajes de embarcaciones y buzos libres y por fuentes bibliográficas (Arceo \& Seijo, 1991) fueron las siguientes: las temporadas de pesca (2007-2008, 2011-2012), mes, captura de especies, unidades de esfuerzo (tiempo efectivo de pesca, número de buzos por viaje en el caso del $\mathrm{BL}$ y número de inmersiones por viaje en el caso del $\mathrm{BH}$ ), variables ambientales (visibilidad, profundidad y ubicación geográfica) y económicas (costo gasolina asociado al viaje de pesca en el caso del $\mathrm{BH}$ y precio promedio por especie).

A través de análisis exploratorios y con base en el conocimiento obtenido durante la observación directa en el campo, se identificaron variables que pudieran tener un efecto de interacción. Por lo tanto, en el proceso de selección de los GAMs, se incluyeron estas interacciones. Para ello se evaluó el nivel de ajuste en los modelos, tanto de los efectos directos de variables individuales separadas, como los efectos de la interacción entre variables. Esto permitió seleccionar las variables del modelo y así evitar efecto de colinealidad de predictores idénticos. De esta manera, se incorporaron en los modelos tres tipos de interacciones: 1) visibilidad * número de buzos por viaje; 2 ) visibilidad * tiempo efectivo; y 3 ) ubicación geográfica (latitud*longitud). Una vez obtenido el modelo con el mejor ajuste para cada especie se procedió a eliminar cada variable o interacción de manera individual, con el fin de calcular su reducción en la desvianza explicada, y poder conocer su aportación a la devianza explicada total.

Patrones temporales de las operaciones de pesca: En este contexto se asume que los pescadores tenderán a modificar sus estrategias para mantener o aumentar sus capturas. Por tanto, se planteó la hipótesis de que el esfuerzo pesquero aumentaría con reducciones en la captura. Esos cambios pueden darse en varias direcciones como cambio en el número promedio de inmersiones que realizaron los buzos, cambios en el tiempo efectivo de buceo (horas) o incluso cambio en la composición de especies. A fin de conocer si los patrones de inmersiones asociados al empleo de $\mathrm{BH}$ cambiaron entre temporadas, se aplicó un análisis de varianza no paramétrico de Kruskal-Wallis $(\mathrm{K}-\mathrm{W})(\mathrm{p}=0.05)$ usando como variable de referencia el número de inmersiones y tiempo efectivo de buceo. Igualmente se esperaría que en la temporada de pesca 2011-2012 los buzos que emplearon BL hayan aumentado el número de buzos por viaje y el tiempo de buceo. Debido a que los buzos que trabajan con BL realizan múltiples inmersiones sucesivas en un área específica seleccionada a priori no resultó práctico analizar estos patrones de inmersiones a la luz de la hipótesis planteada. 
Asignación espacial del esfuerzo pesquero: Con el fin de conocer si la asignación espacial del esfuerzo cambió por temporada y para cada método de pesca, se usó la información registrada en campo de los viajes de pesca de cada uno de los métodos. Esta información fue analizada mediante el programa de información geográfica ArcGIS 9.3 con la extensión "Spatial Analyst".

Para el caso del BL se usó como variable de referencia del número de buzos por viaje considerando los resultados de los modelos GAMs y dado que esta unidad de esfuerzo fue relevante por la función que los grupos de buzos tienen en las faenas de operación (e.g acarreo de captura y cooperación). En el caso del buceo con BH se utilizó el tiempo efectivo de pesca por viaje como la unidad de esfuerzo que mejor explicó la variación de las capturas de pulpo. Con el fin de conocer si la dinámica de la distribución e intensidad de estas unidades de esfuerzo cambia en la escala espacial en contraste con la temporada de pesca, se siguió el procedimiento sugerido por Riolo (2006) en el cual se generan superficies de densidad mediante la función de densidad de kernel. Para el BL la densidad se expresó como el número de buzos por $\mathrm{km}^{2}$ y para el caso de $\mathrm{BH}$ el número de horas de buceo por $\mathrm{km}^{2}$.

\section{RESULTADOS}

\section{Composición de captura entre tempora-} das: Se registraron un total de 13 especies de invertebrados y 19 de vertebrados, de estas 30 fueron retenidas para consumo local, trueque y venta en playa. La categoría definida como "subsistencia" (producto integrado por una mezcla de especies que se retiene para consumo personal) registró un porcentaje promedio anual del $5.6 \%$ y $0.6 \%$ para el BH y BL respectivamente en las dos temporadas de pesca.

Se identificaron seis categorías de captura comunes a ambos métodos de pesca (langosta verde, pulpo, pez loro, pepino, caracol y subsistencia), se observó una mayor diversidad de especies con el uso de BH (Cuadro 1), con altas proporciones para el pulpo Octopus sp, la langosta verde Panulirus gracilis y el pez loro compuesto por las especies Scarus ghobban y $S$. perrico. Las especies de ostión capturadas Spondylus calcifer y $S$. princeps presentaron menor aporte para la temporada 2011-2012. La composición de especies del BHN fue diferente a la composición de especies capturadas durante el día con BH. Para el BHN predominó la captura de pez loro y se observó mayor proporción de pepino de mar (Isostichopus fuscus).

Los pescadores que emplean BH retuvieron mayor captura para fines de subsistencia que los pescadores que emplean BL. Durante los viajes de pesca se comprobó que algunos pescadores que emplearon $\mathrm{BH}$ realizaban inmersiones solo para retener especies para estos fines. Las únicas especies que no fueron destinadas para subsistencia fueron el pepino (I. fuscus) y el mero (Epinephelus itajara).

La contribución de las distintas especies dentro de los viajes para cada método varió con respecto a su aporte en volumen y valor económico en las dos temporadas. Para el caso del BL, la captura de la langosta, el pulpo y el pez loro fue la que tuvo mayor peso dentro de los GLMs. Sin embargo, estas mismas especies contribuyeron en su correspondiente valor a la captura adicionalmente al caracol (Strombus galeatus), que fue la especie de mayor valor económico en las dos temporadas (aproximadamente $14 \$ / \mathrm{kg}$ ) (Cuadro 2). A pesar de que el pulpo fue la especie que registró los mayores volúmenes de extracción para éste método en las dos temporadas, se observó una fuerte diferencia en su aporte al valor de captura debido a su bajo precio promedio por temporada, el cual presentó un aumento de 1.3 dólares para la temporada del 2011-2012 (Fig. 1A, Fig. 1B). La captura de langosta aumentó en la temporada 2011-2012 y para ambas temporadas se ubicó como la especie que aportó los mayores ingresos debido a los altos volúmenes de extracción y a su alto precio.

En la captura de $\mathrm{BH}$, ocho especies mostraron su efecto en los viajes y siete en su valor de captura (Cuadro 2). El pepino registró una considerable disminución en su valor de captura dado el bajo precio pagado en playa. 
CUADRO 1

Composición de captura de los desembarques en Playa Lagarto mediante BL, BH y BHN

en las temporadas 2007-2008 y 2011-2012

TABLE 1

Catch composition of landings from divers that employed BL, BH and BHN in Playa Lagarto in the season 2007-2008 y 2011-2012

\begin{tabular}{|c|c|c|c|c|c|c|}
\hline \multirow{2}{*}{ Nombre común } & \multirow{2}{*}{ Especies } & \multicolumn{2}{|c|}{$\mathrm{BH}(\%)$} & \multirow{2}{*}{$\begin{array}{c}\text { BHN (\%) } \\
2011-2012\end{array}$} & \multicolumn{2}{|c|}{ BL $(\%)$} \\
\hline & & $2007-2008$ & 2011-2012 & & 2007-2008 & 2011-2012 \\
\hline Langosta verde & Panulirus gracilis & 27.5 & 47.6 & 32.1 & 25.9 & 47.2 \\
\hline Pulpo & Octopus sp. & 35.1 & 22.0 & 9.0 & 63.2 & 50.0 \\
\hline Pez loro ${ }^{1}$ & Scarus perrico, Scarus ghobban & 8.6 & 9.5 & 48.2 & 7.3 & 1.2 \\
\hline Subsistencia $^{2}$ & & 6.1 & 5.1 & 3.3 & 0.3 & 1.0 \\
\hline Caracol gigante, caracol caballo & Strombus galeatus, Fasciolaria princeps & 2.7 & 4.7 & 0.6 & 0.8 & 0.5 \\
\hline Pepino de mar & Isostichopus fuscus & 4.2 & 4.1 & 6.8 & 1.6 & 0.1 \\
\hline Ostión, almeja burra & Spondylus calcifer, Spondylus princeps & 11.3 & 3.2 & 0.0 & 0.0 & 0.0 \\
\hline Pargo negro & Lutjanus novemfasciatus & 2.3 & 2.1 & 0.0 & 0.9 & 0.0 \\
\hline Ostra perla & Pinctada mazatlanica & 0.6 & 0.7 & 0.0 & 0.0 & 0.0 \\
\hline Hojarán & Seriola rivoliana & 0.4 & 0.3 & 0.0 & 0.0 & 0.0 \\
\hline Pargo roquero & Hoplopagrus guntheri & 0.1 & 0.3 & 0.0 & 0.0 & 0.0 \\
\hline Pargo coliamarillo & Lutjanus argentiventris & 0.7 & 0.2 & 0.0 & 0.0 & 0.0 \\
\hline Congrio & Brotula clarkae & & 0.1 & 0.0 & 0.0 & 0.0 \\
\hline Cabrilla pana-meña, cabrilla & $\begin{array}{l}\text { Cephalopholis panamensis, } \\
\text { Epinephelus la-briformis }\end{array}$ & 0.4 & 0.0 & 0.0 & 0.0 & 0.0 \\
\hline Mero & Epinephelus itaja-ra & 0.1 & 0.1 & 0.0 & 0.0 & 0.0 \\
\hline Pargo mancha & Lutjanus gutta- tus & 0.1 & 0.0 & 0.0 & 0.0 & 0.0 \\
\hline
\end{tabular}

${ }^{1}$ Epecies comercializadas en filete o lonja.

${ }^{2}$ Incluyeron proporciones tanto de especies comerciales como no comerciales como: caracol caballo (Fasciolaria princeps), almeja reina (Megapitaria aurantiaca), almeja costillada (Periglypta multicostata), concha abanico (Pinna rugosa), caracol cresta de gallo (Strombus peruvianus), chancho cochino (Sufflamen verres), pez lija (Aluterus scriptus), cirujano (Acanthurus xanthopterus), pargo rayado (Lutjanus viridis), tigre (Cirrhitus rivulatus), hojarán amarillo (Seriola lalandi), pámpano (Alectis ciliaris), Cabrilla (Epinephelus labriformis), Cabrilla panameña (Cephalopholis panamensis), roncador (Haemulon scudderi), burrito (Anisotremus interruptus), pez escorpión (Scorpaena histrio).

Especies con altas proporciones de captura en negrilla.

The species with the highest proportions of catches are highlighted in bold.

Para este método, la langosta presentó mayores capturas, seguida por el pulpo y el pez loro. En la temporada del 2011-2012 se observó un incremento en la captura de la mayoría de las especies con respecto a la temporada del 2007 2008, con excepción de la captura de ostión, la que presentó una tendencia opuesta. (Fig. 1C, Fig. 1D).

Composición de especies objetivo entre temporadas: La temporada tuvo un efecto significativo en el promedio de captura por viaje en ambos métodos de buceo (Cuadro 3).
Las mayores diferencias en el uso de BL entre temporadas estuvieron definidas por la captura de pulpo $(\mathrm{F}=0.14, p=0.001)$. Se registró un promedio de $6.3 \mathrm{~kg}$ por viaje para la temporada de pesca del 2007-2008, mientras que para la temporada 2011-2012 fue de $3.1 \mathrm{~kg}$. No se observaron diferencias significativas en la captura de langosta entre temporadas, registrándose $2.5 \mathrm{~kg}$ por viaje en la temporada 2007-2008 y $2.9 \mathrm{~kg}$ en la temporada 2011-2012.

En las capturas que resultaron del uso de $\mathrm{BH}$, las mayores diferencias se encontraron en relación a la langosta $(F=0.108, \mathrm{p}=0.001)$ 
CUADRO 2

Resultados derivados del procedimiento de remuestreo de los MLGs ajustados para la captura y el valor de captura por método de buceo

TABLE 2

Results derived from the resampling procedure of GLMs fitted for catch and catch value for each diving method

\begin{tabular}{|c|c|c|c|c|c|c|c|c|}
\hline \multirow{3}{*}{$\begin{array}{c}\text { Prueba } \\
\text { estadística }\end{array}$} & \multicolumn{4}{|c|}{ Buceo Libre } & \multicolumn{4}{|c|}{ Buceo con Hookah } \\
\hline & \multicolumn{2}{|c|}{ Captura } & \multicolumn{2}{|c|}{ Valor de captura } & \multicolumn{2}{|c|}{ Captura } & \multicolumn{2}{|c|}{ Valor de captura } \\
\hline & Valor LR & Valor $p$ & Valor LR & Valor $p$ & Valor LR & Valor $p$ & Valor LR & Valor $p$ \\
\hline Intercepto & 193.34 & $<0.001 * * *$ & 188.99 & $<0.001 * * *$ & 298.85 & $<0.001 * * *$ & 257.45 & $<0.001 * * *$ \\
\hline Pulpo & 6.25 & $0.016^{*}$ & 4.32 & $0.027 *$ & 137.38 & $<0.001 * * *$ & 76.5 & $<0.001 * * *$ \\
\hline Langosta & 4.67 & $0.009 * *$ & 5.24 & $0.021 *$ & 24.48 & $<0.001 * * *$ & 6.4 & $0.013 *$ \\
\hline Pez loro & 10.34 & $<0.001 * * *$ & 11.97 & $<0.001 * * *$ & 7.65 & $0.006 * *$ & 51.96 & $<0.001 * * *$ \\
\hline Ostión & & & & & 31.15 & $<0.001 * * *$ & 42.49 & $<0.001 * * *$ \\
\hline Subsistencia & 1.35 & 0.249 & 1.22 & 0.29 & 8.31 & $0.004 * *$ & 15.8 & $<0.001 * * *$ \\
\hline Pepino & 2.99 & 0.078 & 0.03 & 0.85 & 20.99 & $<0.001 * * *$ & 0.17 & 0.66 \\
\hline Caracol & 2.82 & 0.093 & 5.98 & $0.010 * *$ & 10.92 & $<0.001 * * *$ & 7.7 & $0.005 * *$ \\
\hline Pargo & & & & & 0.59 & 0.44 & 2.7 & 0.09 \\
\hline Ostra & & & & & 1.13 & 0.27 & 2.5 & 0.1 \\
\hline Significancia & $* * * 0.001$ & $* * 0.01$ & $* 0.05$ & & & & & \\
\hline
\end{tabular}

* significancia estadística. / * statistics significance

que registró $17.7 \mathrm{~kg}$ por viaje en la temporada $2011-2012$ y $10.4 \mathrm{~kg}$ en la temporada $2007-$ 2008. El pulpo registró una disminución en su captura de $4.5 \mathrm{~kg}$ por viaje en la temporada 2011-2012 con respecto a la temporada 2007$2008(F=0.07, \mathrm{p}=0.001)$. La captura del ostión registró una disminución de $2.5 \mathrm{~kg}$ por viaje en la temporada 2011-2012 en comparación a su captura en la temporada 2007-2008 $(F=0.15$, $\mathrm{p}=0.001$ ). Por el contrario, el caracol registró un ligero aumento de $1.9 \mathrm{~kg}$ por viaje en el 2011-2012 con respecto a su captura en la temporada $2007-2008(F=0.03, \mathrm{p}=0.001)$.

Fuentes de variación de las capturas: Gran parte de la variación en la captura total por viaje de pesca entre temporadas para el caso del BL fue explicada por la ubicación geográfica de las faenas de buceo $(24 \%$ de devianza explicada). La captura tuvo una relación directa con los sitios de pesca, el modelo predice que a mayor profundidad y mayor distancia de la costa se registra mayor captura (Fig. 2A). Para el empleo de este método de buceo, la temporada de pesca (efecto del año) fue significativa debido a cambios en la distribución espacial del esfuerzo de pesca de un periodo a otro. La interacción entre la visibilidad y número de buzos por viaje, explicó el $12.3 \%$ de devianza. La captura de pulpo definió un $6.9 \%$ de devianza explicada. Este recurso mostró altos registros de captura y mostró diferencias de captura por viaje entre temporadas Otras variables que contribuyeron en menor proporción a explicar la variabilidad en la captura total entre temporadas fueron el pez loro (1.6\%), el año (1.5\%), el mes (1.3\%) y la langosta (1.2\%). Este modelo GAM logró explicar el $48.8 \%$ de devianza explicada con una puntuación de GCV de 0.3 (Cuadro 4).

El modelo GAM para el BH fue explicado por un mayor número de variables, siendo la ubicación geográfica igualmente importante: la interacción entre la visibilidad y el tiempo efectivo de buceo (11.1\%), mes (8.1\%), ubicación geográfica $(5.3 \%)$ y número de inmersiones por viaje $(4.1 \%)$. Se observó que la captura aumentó al aumentar el tiempo efectivo de buceo a $5 \mathrm{~m}$ de visibilidad de la columna de agua. Sin embargo, el modelo predice que a 


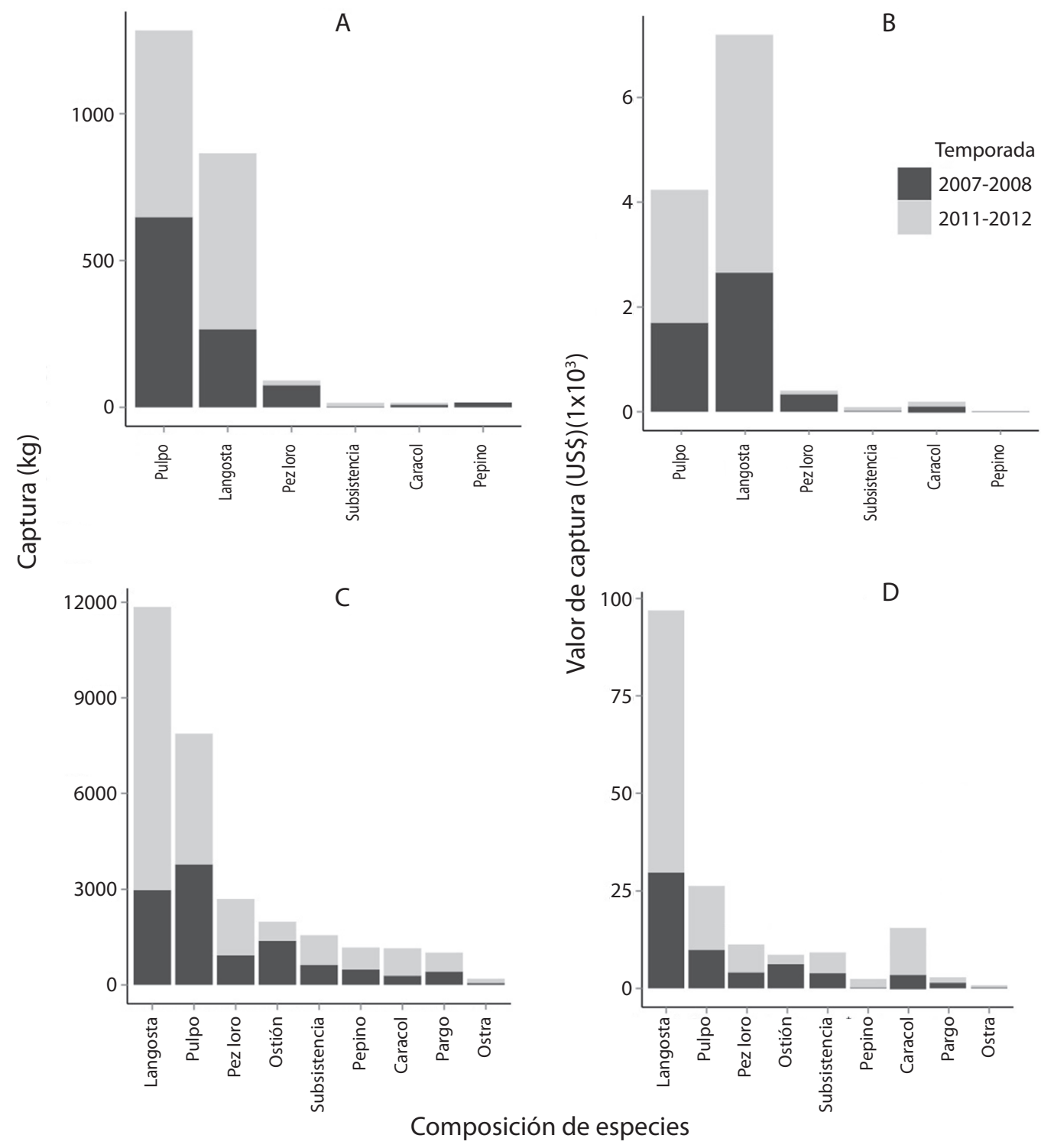

Fig. 1. Captura y valor de captura para el BL (A, B) y para el BH (C, D) en las dos temporadas.* léase subsistencia. Fig. 1. Catch and catch value for $\mathrm{BL}(\mathrm{A}, \mathrm{B})$ and $\mathrm{BH}(\mathrm{C}, \mathrm{D})$ in the two fishing season. * subsistence.

visibilidades mayores a $5 \mathrm{~m}$ se presenta un patrón variable de la captura con respecto a los efectos combinados del tiempo efectivo de buceo y la visibilidad, (e.g. Al aplicarse mayor tiempo efectivo no necesariamente se obtienen mayores capturas) (Fig. 2B). Otras variables con menor peso en la contribución a la devianza entre temporadas fue la profundidad
(2.4\%), el pulpo (1.3\%) y el ostión (1.1\%). Las variables con contribuciones menores $1 \%$ se pueden observar en el cuadro 4. Este modelo logró explicar el $36.7 \%$ de devianza y tuvo una puntuación de GCV de 0.1.

Patrones temporales de las operaciones de pesca: El $59.7 \%$ de viajes que realizaron 


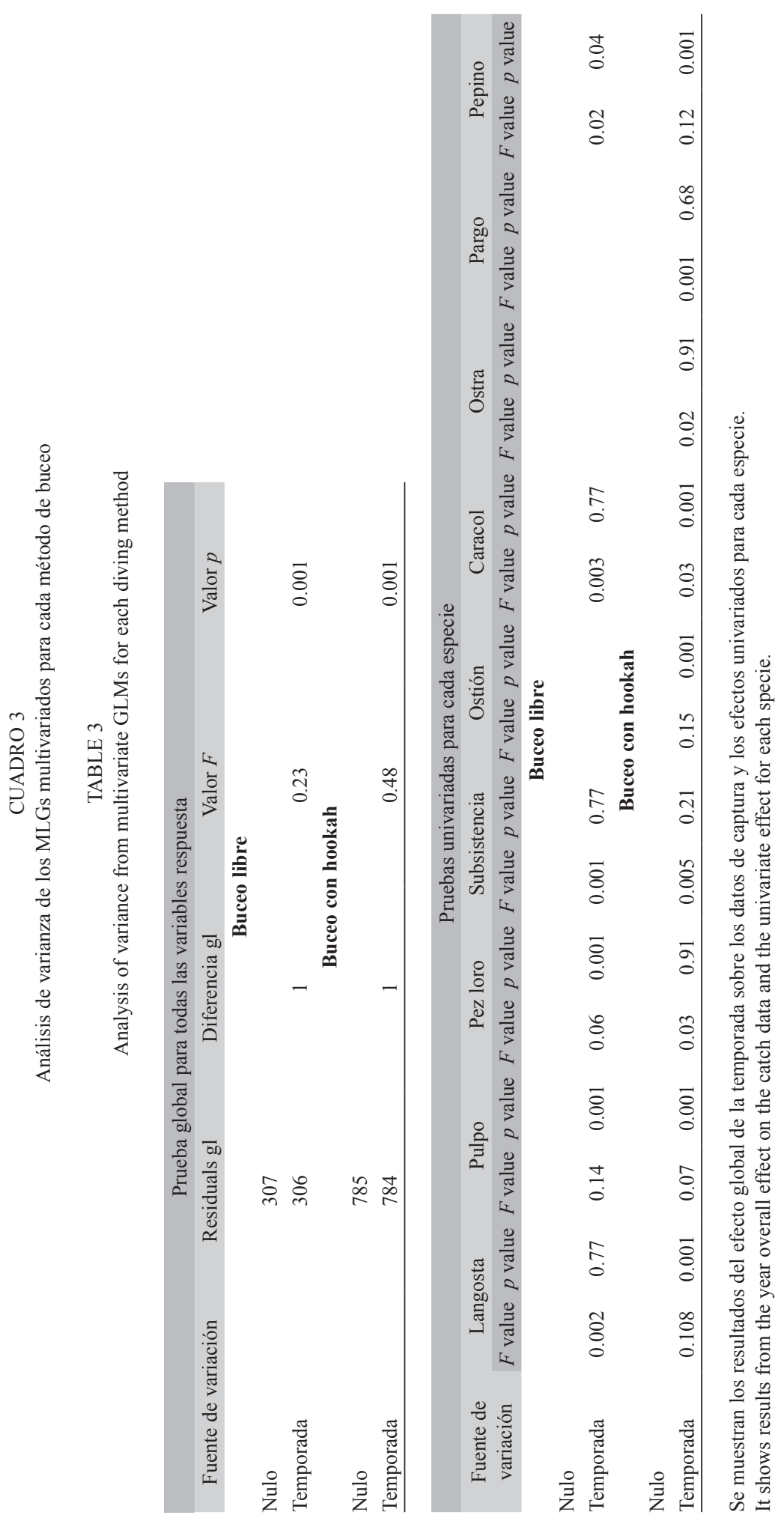




\section{CUADRO 4}

Variables que ajustaron los mejores modelos en la variación de la captura para el total de especies capturadas mediante $\mathrm{BL}$ y BH

TABLE 4

Variables that fit the best models for catch variation for the overall species caught using BL and BH

\begin{tabular}{|c|c|c|c|c|c|c|c|c|}
\hline Parámetro & \multicolumn{4}{|c|}{ Captura (BL) } & \multicolumn{4}{|c|}{ Captura (BH) } \\
\hline Devianza explicada (\%) & & & 48.8 & & & & 36.7 & \\
\hline Intercepto & & & 1.7 & & & & 3.7 & \\
\hline Error estándar & & & 0.08 & & & & 0.005 & \\
\hline$r^{2}$ Ajustado & & & 0.4 & & & & 0.4 & \\
\hline Puntuación GCV & & & 0.3 & & & & 0.1 & \\
\hline Covariables & $\mathrm{gl}$ & Valor $\mathrm{p}$ & Significancia & $\begin{array}{c}\text { Devianza } \\
\text { explicada } \\
\text { parcial (\%) }\end{array}$ & $\mathrm{gl}$ & Valor $\mathrm{p}$ & Significancia & $\begin{array}{c}\text { Devianza } \\
\text { explicada } \\
\text { parcial (\%) }\end{array}$ \\
\hline Temporada & 1 & 0.02 & 0.05 & 1.5 & & & & \\
\hline Mes & 2 & 0.08 & 0.1 & 1.3 & 9 & $<0.001$ & 0.001 & 8.1 \\
\hline Número de inmersiones por viaje & & & & & 3 & $<0.001$ & 0.001 & 4.1 \\
\hline Precio & & & & & 8 & $<0.001$ & 0.001 & 0.2 \\
\hline Costo combustible & & & & & 7 & $<0.001$ & 0.001 & 0.7 \\
\hline Profundidad & & & & & 5 & $<0.001$ & 0.001 & 2.4 \\
\hline Langosta & 2 & $<0.001$ & 0.001 & 1.2 & 5 & $<0.001$ & 0.001 & 0.9 \\
\hline Pulpo & 5 & $<0.001$ & 0.001 & 6.9 & 6 & $<0.001$ & 0.001 & 1.3 \\
\hline Ostión & & & & & 3 & $<0.001$ & 0.001 & 1.1 \\
\hline Pez loro & 2 & 0.02 & 0.05 & 1.6 & & & & \\
\hline Pargo & & & & & 3 & 0.002 & 0.01 & 0.3 \\
\hline Pepino & & & & & 4 & $<0.001$ & 0.001 & 0.7 \\
\hline Subsistencia & & & & & 7 & 0.04 & 0.05 & 0.5 \\
\hline Ubicación geográfica & 6 & $<0.001$ & 0.001 & 24 & 12 & $<0.001$ & 0.001 & 5.3 \\
\hline Visibilidad $\mathrm{x}$ tiempo efectivo & & & & & 18 & $<0.001$ & 0.001 & 11.1 \\
\hline Visibilidad $\mathrm{x}$ número de buzos por viaje & 2 & $<0.001$ & 0.001 & 12.3 & & & & \\
\hline
\end{tabular}
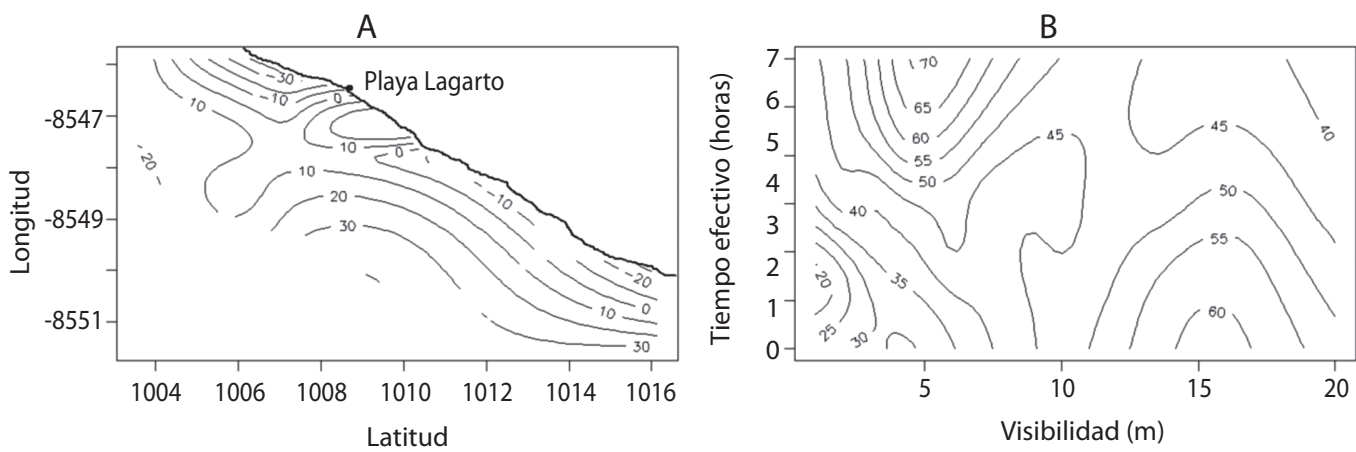

Fig. 2. Los efectos predichos combinados del total de captura por ubicación geográfica para el buceo libre (A) y los efectos predichos combinados del total de captura para el tiempo efectivo y la visibilidad para el buceo con hookah (B) se muestran en los gráficos de isolíneas

Fig. 2. Predicted combined effect on total catch by geographic location from free diving (A) and predicted combined effect on total catch by effective fishing time and water visibility for hookah diving (B) shown by the isoclines plots. 
los buzos que emplearon BL durante las dos temporadas de pesca fueron realizados individualmente, $32.4 \%$ en parejas, $7.2 \%$ en tríos y el $0.7 \%$ en grupos de cuatro. No se encontraron diferencias significativas en el número de buzos por viaje entre temporadas $(\mathrm{K}-\mathrm{W}$, $\mathrm{H}=0.57, \mathrm{p}=0.38$ ) pero si en la frecuencia de viajes de grupos de buzos (K-S, $\mathrm{p}<0.01$ ). Se observó que los buzos realizaron con mayor frecuencia viajes individuales y en parejas en la temporada 2011-2012 con respecto la temporada 2007-2008. Sin embargo, en la temporada 2007-2008 los buzos trabajaron más horas con respecto a la temporada 2011-2012 (K-W, $\mathrm{H}=55.2, \mathrm{p}<0.0001)$ (Fig. 3A).

Para el caso del BH, en la mayoría de viajes se realizaron una inmersión (63.1\%), un 13.3\% de viajes realizaron dos inmersiones, $19.2 \%$ tres inmersiones, $4.1 \%$ cuatro inmersiones y solo un $0.3 \%$ efectuaron cinco inmersiones por viaje (de un día). Además, se encontraron diferencias en el número de inmersiones por viaje entre temporadas $(\mathrm{K}-\mathrm{W}, \mathrm{H}=50.9, \mathrm{p}<0.0001) \mathrm{y}$ en la frecuencia de inmersiones por viaje (K-S, $\mathrm{p}<0.01)$ registrándose una mayor frecuencia de viajes ejecutadas por buzos que realizaron dos y tres inmersiones en la temporada 2011-2012. También se observó que los buzos que emplearon BH en la temporada 2007-2008 bucearon más horas que los buzos que trabajaron en la temporada 2011-2012 (K-W, H=106.7, $\mathrm{p}<0.0001$ ) (Fig. 3B).

\section{Asignación espacial del esfuerzo entre} temporadas: En la temporada de pesca 20072008, los buzos que emplearon BL visitaron sitios distantes como Punta Pargos y Punta los Toros hacia el noroeste, así como playa Frijolar y las Concavas hacia el suroeste. En la temporada 2011-2012 el área de distribución espacial de los buzos disminuyó en un $66 \%\left(3.5 \mathrm{~km}^{2}\right)$. A pesar de las diferencias en la asignación espacial del esfuerzo observadas entre temporadas, hubo una mayor densidad de buzos que operaron cerca del puerto pesquero en ambos casos
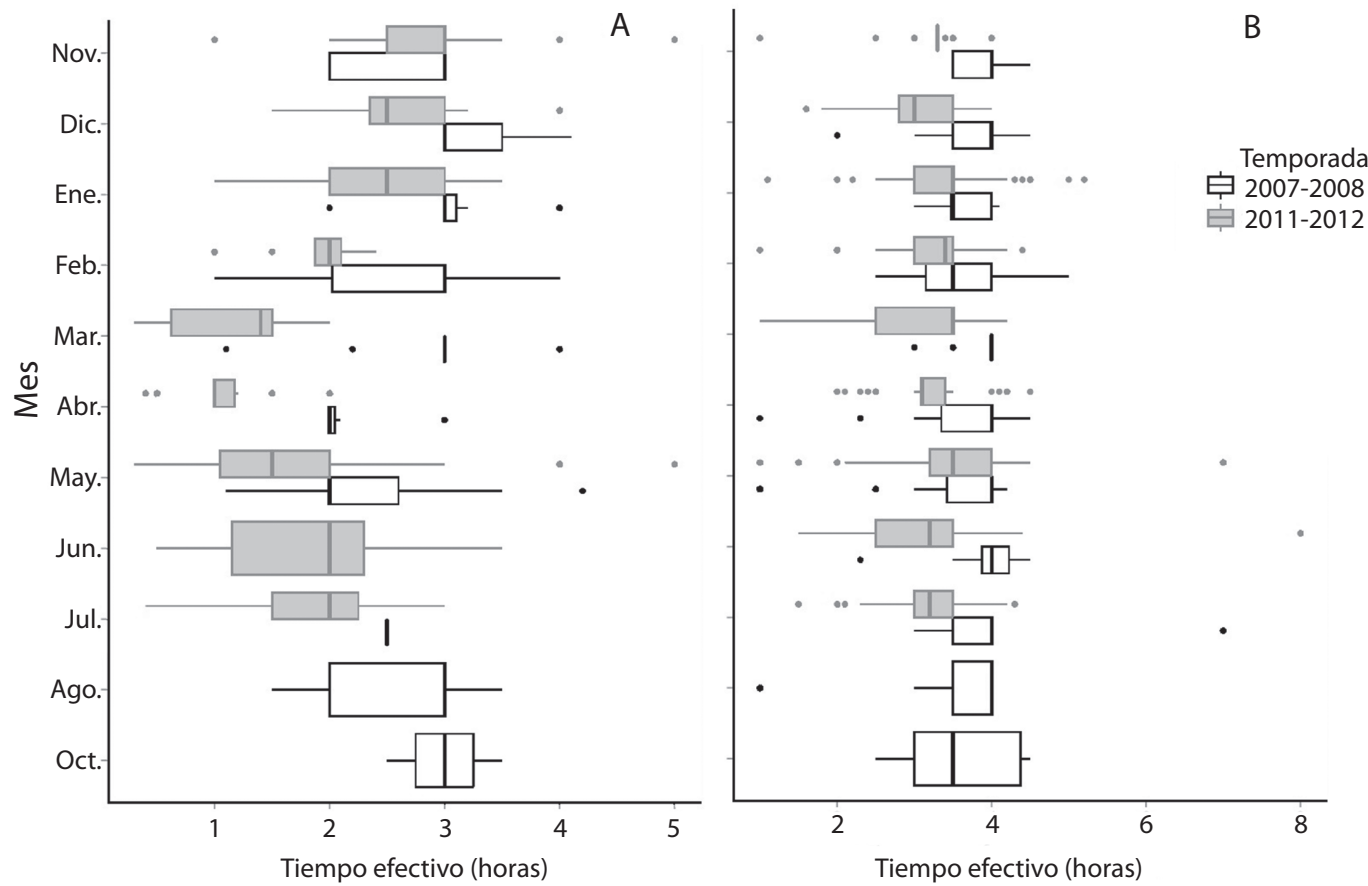

Fig. 3. Tiempo efectivo por mes y entre temporadas para BL (A) y BH (B). Valores atípicos representados por los puntos negros y grises para cada caso.

Fig. 3. Effective time per month and between years for BL (A) and BH (B). Black and grey points show outliers for each case. 


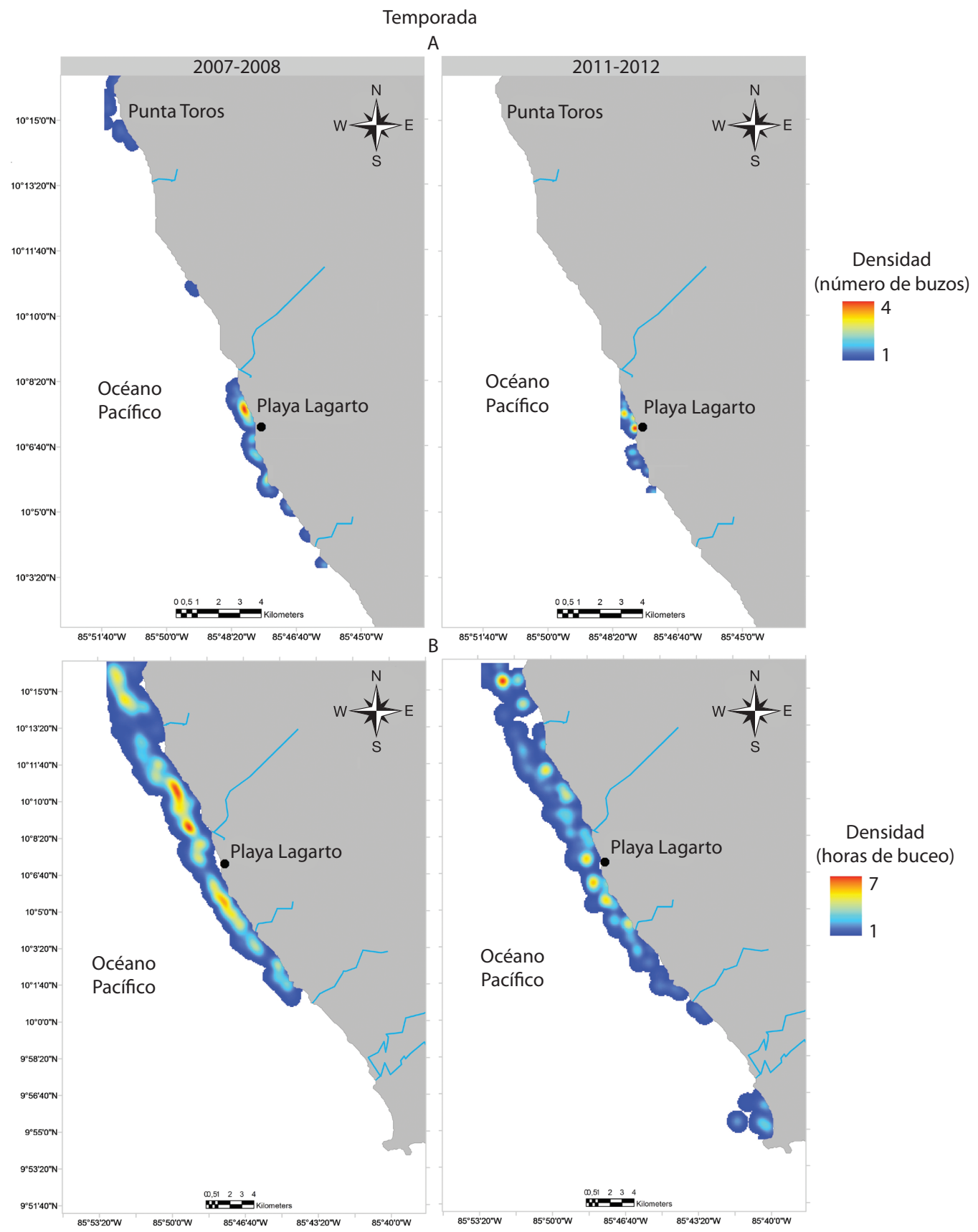

Fig. 4. Distribución especial del esfuerzo de pesca por método de buceo. Densidad de buzos (número de buzos por km²) para el buceo libre (A) y densidad de horas de buceo (número de horas de buceo por $\mathrm{km}^{2}$ ) para el buceo con hookah (B) en las dos temporadas. Altas densidades de esfuerzo se indican en rojo. El puerto pesquero de Playa Lagarto se muestra como referencia.

Fig. 4. Spatial allocation of fishing effort by type of diving method. Density of divers (number of divers per $\mathrm{km}^{2}$ ) for free diving (A) and density of hours (number of diving hours per $\mathrm{km}^{2}$ ) for hookah diving (B) spatially by year. High density of effort is indicated in red. The landing port of Playa Lagarto is indicated as a reference (the main landing port). 
dada la angosta plataforma continental en la zona (Fig. 4A).

En el caso de los buzos que emplearon $\mathrm{BH}$, se observó un aumento de un $12.4 \%\left(7.9 \mathrm{~km}^{2}\right)$ de una temporada a la otra. En la temporada 2011-2012 los buzos registraron densidades promedio de horas de buceo con una tendencia dispersa y en sitios más alejados al puerto pesquero como Punta Guiones al extremo sureste. Para esta temporada las mayores densidades de horas de buceo se dieron en sitios cercanos al puerto pesquero y con mayor intensidad al extremo noroeste, cerca del límite del área marina protegida del Parque Nacional Marino Las Baulas. En la temporada 2007-2008 las mayores densidades de esfuerzo (horas de buceo) se dieron hacia el noroeste en los sitios conocidos como Playa Negra y Punta Pargo, al igual que en los sitios cercanos al puerto pesquero (Fig. 4B).

\section{DISCUSIÓN}

Las diferencias encontradas en la composición de especies entre temporadas en el empleo de BL pudieran ser explicadas por la disminución en su área de distribución en el 2011-2012 con respecto a su distribución en el 2007-2008. Además, en la última temporada de pesca los buzos que emplearon el BL bucearon menos horas. Sin embargo, la disminución considerable en la captura de pez loro en la última temporada de pesca pudo ser causada por la gran presión de pesca sobre el pez loro que ejerce el $\mathrm{BH}$ y el BHN en parches de arrecife cercanos a los sitios de pesca donde opera el BL. Esta interacción entre los métodos de buceo podría ser considerada como una interdependencia tecnológica, donde el $\mathrm{BH}$ y el $\mathrm{BHN}$ remueven parte de captura potencial en áreas cercanas a los sitios donde opera el BL. Interdependencias tecnológicas entre métodos de buceo no han sido documentadas en la literatura, pero si entre flotas. Por ejemplo, Horta \& Defeo (2012) encontraron efectos negativos de las actividades de la flota industrial sobre la capturas de la flota artesanal mediante el uso de un índice de interdependencia tecnológica que estima el nivel de traslape entre áreas de pesca.

Se debe considerar el efecto que puede tener el empleo de $\mathrm{BH}$ y $\mathrm{BHN}$ sobre especies de poca movilidad como el ostión ( $S$. calcifer y $S$. princeps), el pepino (I. fuscus) y el caracol ( $S$. galeatus). En el área de estudio existe una medida de regulación que prohíbe la captura de las últimas dos especies, sin embargo no existe vigilancia que permita el cumplimiento de esta medida. Adicionalmente, la captura de ostión no se encuentra regulada debido a la inexistencia de estudios sobre la biología de las especies $S$. calcifer y $S$. princeps en la región. Estas especies al presentar desove de difusión pueden ser susceptibles a colapsos repentinos cuando se incrementa la destrucción de su hábitat o la presión de pesca disminuye la población a bajas densidades que impide una adecuada fertilización y el subsecuente reclutamiento (Karlson \& Levitan, 1990). Ejemplo de esto es el collapso de varias industrias productoras de ostra perla en mundo (Donkin, 1998) y el abalon blanco en California (Hobday, Tegner, \& Haaker, 2001).

Si bien la pesquería de especies bentodemersales asociada al buceo en Playa Lagarto exhibe un complejo patrón que incluye una mezcla amplia de especies, el empleo de ambos métodos de buceo coincide en la pesca de seis categorías de especies comunes. Sin embargo, con base en los resultados de los GLMs solamente tres se podrían considerar como especies objetivo comunes entre ambos métodos en las dos temporadas: la langosta verde, pulpo y las dos especies de pez loro (procesado en filete). Estas son especies que se comercializan y también se consumen localmente. Pesquerías asociadas a comunidades rurales en países subdesarrollados muestran patrones similares. Por ejemplo, Kronen (2004) reportó en Tonga que una proporción de la captura era consumida por los pescadores o compartida con sus allegados y no entraba en el mercado. La captura que entraba en el mercado era un resultado de la necesidad de dinero en efectivo y no una respuesta a la demanda del mercado. En nuestro caso de estudio, la tendencia a la retención de 
especies para fines de seguridad alimentaria se dio en mayor proporción bajo el empleo de $\mathrm{BH}$ y BHN y era utilizada para llevar alimento a las familias de los propios buzos.

La existencia de este tipo de prácticas de subsistencia es común en contextos socioeconómicos de países subdesarrollados donde otras opciones económicas no están disponibles (Sadovy, 2005). En general, esta región presenta graves problemas de pobreza y desempleo. Los resultados de la última Encuesta de Hogares realizada por el Instituto Nacional de Estadísticas y Censos de Costa Rica (INEC, 2012) afirman que la provincia de Guanacaste es la segunda más pobre de Costa Rica y la que refleja mayores índices de desempleo por insuficiencia de horas laborales.

Es importante resaltar que la degradación de ecosistemas costeros provocada por la pesca u otros factores como la contaminación, cobra mayor relevancia en contextos donde aspectos de subsistencia y comerciales se combinan. En estos contextos la degradación de parches de arrecife no solo representa la pérdida ecológica en términos de biodiversidad sino también una severa pérdida económica (Sadovy, 2005) y limita el acceso de alimento de origen marino, lo que afecta a comunidades que viven por debajo del umbral de la pobreza (Moberg \& Folke, 1999; Béné, 2003).

Los resultados de los GAMs mostraron que los factores con mayor peso en la captura de especies bento-demersales mediante el empleo de $\mathrm{BL}$ y $\mathrm{BH}$ entre temporadas fueron aspectos ambientales, espacio-temporales y del pescador, estos últimos representados por las unidades de esfuerzo. El poco peso que tuvieron las variables económicas en la variación de captura promedio total por viaje de pesca en los GAMs reafirma lo encontrado por otros estudios que han analizado pesquerías de la misma naturaleza. Por ejemplo, Daw (2008) encontró que en las decisiones sobre la elección de sitios de pesca de los pescadores que capturan langosta en Nicaragua, se tienen en cuenta una compleja gama de aspectos más que la simple relación costo-captura que ha sido muy utilizada en análisis económicos reduccionistas en pesquerías. En nuestro caso, el poco efecto de las variables económicas indica que las propiedades de los métodos de pesca implican bajos costos de operación tal como lo reporta Naranjo (2010) al estudiar algunos aspectos económicos de la pesca de langosta con el empleo de los métodos de buceo analizados. Además, el contexto socio-cultural puede estar influyendo en la conducta de los pescadores y por ende en el esfuerzo, tal como señalan varios autores (van Putten et al., 2012; Pollnac \& Poggie, 2008). Lo anterior podría explicar en parte, la proporción de varianza no explicada dentro de los modelos en los dos métodos de buceo.

El resultado del GAM en el empleo de $\mathrm{BH}$ mostró que el factor de más peso en la explicación de la variación de la captura promedio total por viaje de pesca entre temporadas fue el mes, dado el aumento y disminución en la captura de algunas especies en los meses del 20112012 con respecto a su captura en los meses del 2007-2008. También la mayor cantidad de inmersiones a altas visibilidades, en sitios más profundos y alejados del puerto pesquero, tuvo un efecto positivo importante que explicó parte de la variación de la captura entre temporadas e hizo aumentar la captura de langosta en el 2011-2012. Lo anterior indica que en la determinación de la elección de los sitios de buceo los pescadores toman en cuenta en primera instancia aspectos ambientales que son evaluados desde el puerto de pesca o durante la faena de operación in situ, más que los costos asociados al viaje de pesca entre temporadas, aunque la visita a sitios alejados tuvo su efecto al explicar la variación de captura promedio total por viaje de pesca pero en menor proporción.

Los estudios de Moses et al. (2002) y Lopes y Begossi (2011) señalan que las capturas en pesquerías artesanales de arrastre, red agallera, cuerda y buceo se ven influenciadas en el corto plazo por factores hidro-climáticos estacionales. Béné y Tewfik (2001) igualmente reportan cambios en los patrones de los buzos en función de condiciones ambientales y la experiencia de los buzos; los autores también reportan el efecto del mercado en los patrones de pesca. Esto coincide con los resultados 
encontrados en nuestro caso de estudio, sin embargo existió una diferencia en la forma de utilización de los recursos en Playa Lagarto, ya que una proporción de la variación de la captura en el empleo de $\mathrm{BH}$ fue explicada por la captura que se destina a subsistencia, lo cual es reflejo de aspectos socio-económicos y culturales que definen el contexto en que se desarrolla la actividad.

Dado que el empleo del BL implica menores costos de operación (Naranjo, 2010) y es afectado por aspectos oceanográficos, las variables económicas como el precio y los costos de operación no tuvieron un peso importante en el GAM. Para este método las diferencias en los sitios visitados mostraron su efecto en la explicación de la variabilidad de la captura promedio por viaje dada la disminución del área de operación que se dio entre temporadas. Además, el aumento de captura asociado con el aumento del número de buzos por viaje en escalas de visibilidad alta entre temporadas se relacionó con aspectos de cooperación durante la faena de pesca que permitieron mejorar la búsqueda sobre parches de arrecife, acarreo de la captura y en general mayor confianza que representó el buceo con otros compañeros. Uno de los pocos estudios donde se ha analizado el esfuerzo en pesquerías asociadas al buceo encontró similares resultados, el aumento de los grupos de buzos influyó positivamente en un incremento en la captura de peces ornamentales en Hawaii (Stevenson et al., 2011).

Los modelos obtenidos permitieron identificar los factores que contribuyen a la variabilidad en las capturas para cada método de buceo entre temporadas, así mismo facilitaron la identificación de estrategias de captura asociadas con las unidades de esfuerzo y su relación con las variables espaciales, temporales y ambientales. En el caso del BL el número de buzos en operación parece incidir fuertemente en el proceso de captura cuando operan a visibilidades altas y para el $\mathrm{BH}$ el número de inmersiones por viaje representó un uso óptimo de los sitios de pesca. Debido a que la captura por unidad de esfuerzo (CPUE) está compuesta por la compleja relación entre la captura (abundancia de recurso) y el esfuerzo (reflejo de la conducta del pescador) (Voges et al., 2005), los resultados sugieren que estas unidades de esfuerzo podrían ser utilizadas para construir índices de esfuerzo y abundancia más adecuados para la evaluación del recurso y la pesquería.

El BHN representó una práctica de pesca que se podría considerar como una estrategia que aplican los pescadores que emplean $\mathrm{BH}$ para aumentar o mantener la captura de especies como la langosta, el pez loro y el pepino de mar. Ante la disminución en la captura de especies de alto valor comercial como la langosta $P$. gracilis, en las áreas de pesca tradicionales, los pescadores desarrollaron esta estrategia oportunista basada en su conocimiento empírico de la distribución espacial de especies de hábitos crípticos, que durante la noche salen de sus refugios para realizar actividades de forrajeó o reproducción (P.gracilis, I. fuscus) y otras se resguardan en parches de arrecife $(S$. ghobban y $S$. perrico).

La estrategia oportunista representada por el empleo del BHN hizo que se mantuviera estable la captura de pez loro de una temporada de pesca a otra. El BHN concentra su esfuerzo en parches de arrecife donde las especies $S$. ghobban y $S$. perrico acostumbran pernoctar, donde son capturadas fácilmente con el empleo de arpones. La alta vulnerabilidad a la captura que presentan estas especies durante la noche provocó un cambio en la composición de especies desembarcadas durante los viajes del BHN desplazando a otras especies de importancia como la langosta y el pulpo. De incrementarse el esfuerzo mediante el empleo del BHN existiría un mayor riesgo en afectar la población dada la incertidumbre existente sobre su dinámica poblacional. En países donde los pescadores han aplicado este tipo de técnicas de captura (Sabetian, 2010) se ha reportado un rápido descenso en las descargas. Esta disminución poblacional ha provocado cambios ecosistémicos que se reflejan en un aumento en el crecimiento de alga filamentosa y vacíos tróficos que han sido llenados por organismos ciguatera (Gillett \& Moy, 2006). Esto hace que el sistema adquiera condiciones no aptas para 
el reclutamiento de especies coralinas resultando en una pérdida de la complejidad estructural de los arrecifes (Graham et al., 2007). Dada la función que tienen estos herbívoros en los ecosistemas costeros resulta urgente imponer alguna medida específica para regular su captura.

Aunado a la estrategia del BHN los pescadores que emplearon $\mathrm{BH}$ aplicaron otras estrategias de captura que se deducen del resultado de los GAMs y de los análisis de la distribución espacial y temporal del esfuerzo: i) abarcaron mayor área en su distribución espacial del esfuerzo, bucearon en sitios más alejados en la última temporada de pesca lo que tuvo un ligero efecto en los costos; ii) realizaron mayor cantidad de inmersiones por viaje lo que indica que visitaron más sitios en búsqueda de mayores capturas; iii) bucearon en zonas más profundas. Estas estrategias promovieron cambios en los rendimientos y en la composición de la captura y permitieron compensar pérdidas económicas provocadas por la disminución de recursos como el ostión y el pulpo. Para el caso del BL se destaca la estrategia de formación de grupos que se asocia con aspectos de cooperación durante la faena de pesca y minimización del riesgo al enfrentar condiciones oceanográficas adversas.

Pesquerías multi-específicas asociadas a métodos de buceo en América Latina ejercen alta presión de pesca sobre recursos bentodemersales, no solo por su alto valor comercial sino como seguridad alimentaria (Defeo \& Castilla, 2005; FAO, 2006; Daw, 2008). En este tipo de pesquerías es común que los pescadores perciban disminución en los recursos y como efecto compensatorio aumenten el esfuerzo efectivo. En algunos casos, esta disminución no puede ser percibida en las descargas sin existir detallada información de los cambios en la conducta, tecnología y distribución espacial del esfuerzo de pesca (Daw, Robinson, \& Graham, 2011). Los resultados observados en este estudio muestran que los patrones espaciotemporales del esfuerzo pesquero pueden cambiar en el tiempo ante cambios en las capturas o las condiciones del ambiente. Estos patrones se podrían considerar como estrategias que aplican los pescadores para maximizar o al menos mantener las capturas de las especies comerciales más importantes. Estas estrategias estuvieron definidas en un ambiente oceanográfico cambiante, en donde variables ambientales así como factores operacionales contribuyeron a la variabilidad en las capturas.

Tradicionalmente, estas pesquerías artesanales de pequeña escala se les atribuye un bajo impacto en los ecosistemas de los cuales dependen (Hawkins \& Roberts, 2004) y el esfuerzo pesquero se considera muy bajo como para tener efectos importantes sobre los stocks. Sin embargo, como se comprueba en este estudio, cambios en las estrategias de pesca pueden ser causadas por varios factores, como la disminución de la captura de especies de poca y mediana movilidad representadas por el ostión y el pulpo, dadas las diferencias en la captura por viaje registradas entre temporadas principalmente en el caso del BH. Naranjo (2011) encontró en esta área sobrepesca en el crecimiento para el caso de la langosta verde $P$. gracilis, lo que pone en riesgo la sostenibilidad del stock de esta especie a largo plazo. Por lo anterior, se confirma que en estas pesquerías las estrategias y prácticas de pesca deben ser evaluadas ya que ayudan a cuantificar su impacto sobre los recursos e implementar medidas específicas de manejo.

Se ha observado que la distribución por parches de algunas especies bento-demersales hace que el esfuerzo pesquero no sea al azar y que se aplique en áreas de altas densidades (Navarte, González, \& Filipo, 2007). En nuestro estudio esto fue confirmado en las diferencias de la distribución espacial entre temporadas en el empleo de ambos métodos y en los resultados de los GAMs al registrarse un efecto significativo del factor espacial en la explicación de la variación de la captura, lo que indica que para ambos métodos de buceo la selección del sitio es un factor crucial en el éxito de captura.

Esto se podría interpretar bajo el concepto de perfiles de concentración desarrollado por Clark (1982), quién analiza las implicaciones de la distribución espacial de pescadores con 
respecto a su CPUE. Este concepto asume que los pescadores poseen información sobre la ubicación y densidad de los stocks, o la obtienen durante el proceso de operación, y se espera que inicien su proceso de captura sobre parches de alta concentración, hasta alcanzar un nivel bajo de CPUE que los desalienta y obliga a movilizarse hacia otros sitios (Morsan, 2007). Este patrón de elección de sitios parece coincidir con los patrones espaciales de distribución del esfuerzo encontrados de una temporada a otra, con una tendencia más marcada para el caso del $\mathrm{BH}$, en donde los patrones de distribución espacial cambiaron hacia sitios más alejados y zonas más profundas.

Se observó una tendencia diferente en el empleo del BL, los pescadores bucearon menos horas y disminuyeron su distribución espacial en la temporada de pesca 2011-2012 y como resultado la captura de pulpo registró una disminución. Sin embargo, la frecuencia de viajes se incrementó en esta última temporada lo que indica que a pesar que la intensidad del esfuerzo aumento en zonas cercanas al puerto de pesca la captura de pulpo disminuyó. Esto demuestra que la disponibilidad de las especies puede ser explicada por la localización de los sitios de pesca, con un alto peso en el caso de especies de hábitos crípticos como la langosta verde y el pulpo. Se ha demostrado que las especies de invertebrados tienen distribuciones discontinuas, con camas, enclaves o concentraciones de densidades variables en función del tipo de sustrato, corrientes, profundidad y otras características ambientales (Navarte et al., 2007).

Coincidentemente, se observó que el esfuerzo de los buzos en ambos métodos se concentra en parches de arrecife con características estructurales y ecológicas que permiten la presencia de importantes agregaciones de las principales especies objetivo de pesca y evitaban bucear en fondos arenosos. Estos sitios fueron sitios conocidos por los pescadores y destacaron aquellos cercanos a desembocadura de ríos, zonas de fuerte oleaje, sitios profundos, y los sitios ubicados en la zona adyacente al área marina protegida del Parque
Nacional Marino las Baulas. Morsan (2007) reporta similares patrones para la captura de la almeja púrpura en Argentina y Ríos-Lara, Salas, Javier, y Ayora (2007) en el caso de la langosta espinosa $(P$. argus) de las costas de Yucatán, México. La elección de estos sitios por parte de los pescadores de Playa Lagarto se dio antes del zarpe o durante la faena operacional una vez evaluadas las condiciones oceanográficas. En la pesquería de langosta con trampas en Baja California, la distribución especial del esfuerzo de pesca toma en cuenta estos factores ambientales al igual que aquellos sitios que han registrado altas capturas previas (Shester, 2010).

La capacidad que tienen los pescadores en modificar su conducta y así cambiar el método de pesca, la especie objetivo o viajar a mayores distancias indica que los manejadores deben de considerar estos cambios potenciales en la conducta de los pescadores cuando se evalúa el impacto de una estrategia de manejo sobre el recurso pesquero. Por ejemplo, después de una veda a la pesca con redes en Florida se encontró que la mayoría de los pescadores cambiaron los métodos de captura o la especie objetivo con el fin de continuar pescando (Smith, Jacob, \& Jepson, 2003). Debido a la disminución de las poblaciones en Nueva Escocia, se comprobó que los pescadores pescaban más lejos de sus puertos de pesca (Binkley, 2000). Como resultado del impacto potencial de estos cambios, los administradores pesqueros pueden beneficiarse de la investigación a priori de la conducta del pescador para determinar la probable eficacia y el impacto global de las nuevas estrategias.

Dada la gran variedad de especies involucradas en esta pesquería es necesario identificar estrategias de manejo específicas dirigidas a cada grupo (moluscos y crustáceos) enfocándose, por ejemplo, en el ciclo de vida, estación reproductiva, distribución espacial y disponibilidad de biomasa. Planes de manejo deben considerar la naturaleza multi-específica de esta pesquería y las capacidades de los pescadores en cambiar sus estrategias de pesca según la disponibilidad del recurso, factores 
ambientales, exigencias del mercado y aspectos socio-culturales. Es necesario incorporar estos componentes en un mismo marco holístico de manejo que integre la complejidad de estos sistemas pesqueros y permita la construcción de capacidades para el manejo. Aproximaciones de este tipo han sido propuestas recientemente en estudios sobre pesquerías artesanales (e.g. Plagányi et al., 2013; Ahmed, Rahman, Bunting, \& Brugere, 2013).

Mediante el análisis del esfuerzo y las capturas asociadas a los métodos de buceo durante dos temporadas de pesca se pudieron identificar diferencias en los patrones de la captura y esfuerzo de pesca a través del tiempo y espacio. Esta información es crucial para el manejo de pesquerías. Por ejemplo, la baja actividad de buceo registrada durante la temporada de lluvias podría justificar una veda temporal sobre la actividad pesquera y así evitar un fuerte impacto socio-económico lo que permitiría mejorar la disponibilidad de especies bento-demersales a mediano plazo. Además, los patrones espacio-temporales del esfuerzo identificados en este estudio podrían ser utilizados para establecer herramientas de manejo espacial explicitas como zonas de exclusión pesquera, y cierres espaciales temporales. También esta información resulta útil para identificar necesidades de investigación hacia las especies más susceptibles a ser sobreexplotadas como por ejemplo el ostión, el pepino y el caracol.

En general, existen grandes deficiencias en el manejo de los recursos pesqueros en el Pacifico Norte de Costa Rica. Las medidas de manejo para los recursos capturados incluyen: i) talla mínima de captura para la langosta $P$. gracilis y no captura de hembras ovígeras, ii) límites al esfuerzo pesquero (licencias, carnet de buceo), iii) prohibición de captura y comercialización de pepino I. fuscus y caracol $S$. galeatus. Estas medidas no han sido aplicadas sistemáticamente en los puertos pesqueros del Pacífico Norte por lo cual que no se descarta que la disminución de la captura de ostión y pulpo encontrada en este estudio en Playa Lagarto pudiera estar asociada al régimen de pesca sostenido durante varias décadas. Además, factores como el alto precio de algunas especies bento-demersales, la ausencia de alternativas de trabajo para los habitantes de las comunidades costeras, el débil compromiso de los pescadores de los puertos pesqueros con objetivos de manejo y la ausencia de estrategias de manejo sostenible hace que se promueva la sobreexplotación.

Debido a la ausencia de información biológica sobre el estado de stock de ostión, pulpo y pez loro se recomienda aplicar el enfoque precautorio e implementar las siguientes medidas para controlar una potencial sobreexplotación: a) vedar la captura de ostión, b) aplicar la veda de langosta recomendada por Naranjo (2012) y que adicionalmente abarque al pulpo y pez loro, c) conviene aplicar paralelamente a las vedas algún tipo de compensación o subsidio para los pescadores, d) realizar estudios biológico-pesqueros que permitan la captura estacional de estas especies (ostión, pulpo y pez loro) con el fin de promover el uso sostenible de estos recursos. Adicionalmente, es necesario que las instituciones gubernamentales encargadas de administrar los recursos pesqueros apliquen y vigilen las regulaciones existentes y se fomente la participación activa de las comunidades en el manejo de los recursos que asegure la sostenibilidad a largo plazo. Resulta indispensable implementar acciones dirigidas a la construcción de la capacidad en el manejo que estén promovidas tanto por el sector público como privado (ONG's) que cubran aspectos como: capacitación laboral, gobernanza, acceso a créditos y subsidios, mejoramiento de la infraestructura portuaria y post-captura, problemas sanitarios (e.g contaminación), y sociales (e.g drogas, alcoholismo) entre otros.

\section{AGRADECIMIENTOS}

Agradecemos a los pescadores de Playa Lagarto, quienes hicieron posible la realización de este estudio. Igual agradecemos a los intermediaros $\mathrm{y}$ a todos aquellos que de una $\mathrm{u}$ otra forma colaboraron en esta investigación. Por su apoyo en la estadística agradecemos 
a David Warton en la Universidad de Whales en Australia y a Manuel Espínola en la Universidad Nacional en Costa Rica. Este trabajo forma parte de la tesis doctoral del primer autor en el CINVESTAV U. Mérida. Se agradece al CONACYT y ALNA S. A por el apoyo financiero.

\section{RESUMEN}

Las pesquerías artesanales asociadas a métodos de buceo son fuente de ingreso, empleo y seguridad alimentaria de zonas costeras en muchos países. Los altos precios y la poca movilidad de especies bénticas capturadas por buceo enfrentan retos cada vez mayores en su aprovechamiento y manejo, esto demanda un entendimiento integral de estas pesquerías en diferentes contextos, incluyendo la dinámica espacial y temporal del esfuerzo pesquero, las artes y las especies. En el presente estudio se busca elucidar si existen diferencias en las estrategias de pesca desarrolladas por pescadores que emplean buceo en dos modalidades (hookah y buceo libre). También se busca conocer si se desarrollan adaptaciones de estas estrategias en el tiempo, bajo qué condiciones y el posible efecto de las estrategias sobre las capturas. Para ese fin se analizó información detallada de las operaciones de pesca de embarcaciones artesanales que operan en la costa del océano Pacífico de Costa Rica durante dos temporadas de pesca. Se analizó información obtenida a bordo de embarcaciones como: sitio de pesca, composición de especies, tiempo de pesca, número de inmersiones por viaje, entre otros; y se aplicaron entrevistas a buzos al momento de las descargas para obtener información de precios, el volumen de las especies capturadas y forma de operación de los pescadores. Para el buceo con hookah se obtuvo una muestra del $69.3 \%$ del total de viajes en las dos temporadas de pesca y en el caso del buceo libre se obtuvo una muestra del $41.9 \%$ del total de viajes en las dos temporadas de pesca estudiadas (20072008 y 2011-2012). Se identificaron más de 15 especies en cada temporada de pesca, dominaron tres categorías en ambas temporadas pero con diferencias en las proporciones obtenidas en cada caso: langosta (Panulirus gracilis), pulpo (Octopus sp) y pez loro (Scarus perrico y S. ghobban). Resalta un componente importante de captura retenida por los pescadores para consumo personal, incluyendo especies de alto valor comercial. Se identificaron cambios en las estrategias de pesca asociadas a cambios en las capturas y cambios en la asignación espacial del esfuerzo pesquero de una temporada de pesca a otra, definidas por la adición de buceo nocturno, aumento en el número de inmersiones de una temporada a otra y procesos de cooperación en las faenas de pesca de buzos que usan buceo libre. Se discute las potenciales causas de los cambios en las estrategias de pesca observadas y se derivan implicaciones para el manejo que permiten la sostenibilidad de estas pesquerías a largo plazo.

Palabras clave: Costa Rica, esfuerzo de pesca, estrategias de pesca, pesquería de buceo artesanal, manejo sostenible, Modelos Aditivos Generalizados.

\section{REFERENCIAS}

Alfaro-Shigueto, J., Mangel, J. C., Pajuelo, M., Dutton, P., Seminoffc, J., Brendan, J., \& Godle, J. (2010). Where small can have a large impact: Structure and characterization of small-scale fisheries in Peru. Fisheries Research, 106, 8-17.

Ahmed, N., Rahman, S., Bunting, S., \& Brugere, C. (2013). Socio-economic and ecological challenges of small-scale fishing and strategies for its sustainable management: A case of study of the Old Brahmaputra River, Bangladesh. Singapore Journal of Tropical Geography, 34, 86-102. doi:10.1111/sjtg.12015

Andrew, N., Béné, C., Hall, S., Allison, E., Heck, S., \& Ratner, B. (2007). Diagnosis and management of small-scale fisheries in developing countries. Fish and Fisheries, 8, 227-40.

Arceo, P. \& Seijo, J. C. (1991). Fishing effort analysis of the small-scale spiny lobster (Panulirus argus) fleet of the Yucatan shelf. FAO Fisheries Report, 431, 59-74.

Béné, C. \& Tewfik, A. (2001). Fishing effort allocation and fishermen's decision making process in a multispecies small-scale fishery: analysis of the conch and lobster fishery in Turks and Caicos Islands. Human Ecology, 29, 157-186.

Béné, C. (2003). When fishery rhymes with poverty: a first step beyond the old paradigm on poverty in smallscale fisheries. World Development, 31, 949-975.

Binkley, M. (2000). "Getting by" in troubled times: coping with the fisheries crisis. Women's Studies International Forum, 23, 323-332.

Caddy, J. F. \& Defeo, O. (2003). Enhancing or restoring the productivity of natural populations of shellfish and other marine invertebrate resources. (FAO Fisheries Technical Paper. No. 448). Rome: Food and Agriculture Organization of the United Nations.

Clark, C. W. (1982). Concentration profiles and the production and management of marine fisheries. In W. Eichhorn, R. Henn, K. Neumann, \& W. Shephard (Eds.), Economic theory of natural resources (pp. 97-112). Berlin, Heidelberg: Springer-Verlag.

Daw, T. M. (2008). Spatial distribution of effort by artisanal fishers: Exploring economic factors affecting the lobster fisheries of the Corn Islands, Nicaragua. Fisheries Research, 90, 17-25. 
Daw, T. M., Robinson, J., \& Graham, N. A. (2011). Perceptions of trends in Seychelles artisanal trap fisheries: comparing catch monitoring, underwater visual census and fishers' knowledge. Environmental Conservation, 38, 75-88.

Defeo, O. \& Castilla, J. C. (2005). More than one bag for the world fishery crisis and keys for co-management successes in selected artisanal Latin American shellfisheries. Reviews in Fish Biology and Fisheries, 15, 265-283.

Donkin, R. A. (1998). Beyond price: pearls and pearl fishing: origins to the age of discoveries. Philadelphia, U.S.A.: American Philosophical Society.

Edgington, E. S. \& Onghena, P. (2007). Randomization Tests. Florida, U.S.A.: Chapman \& Hall.

Eriksson, H., de la Torre-Castro, M., \& Olsson, P. (2012). Mobility, Expansion and Management of a MultiSpecies Scuba Diving Fishery in East Africa. PLoS ONE, 7(4).

FAO. (2006). Informe del quinto Taller Regional sobre la Evaluación y la Ordenación de la Langosta Común del Caribe. Mérida, Yucatán, México. (FAO, Informe de Pesca No. 826). Roma: Food and Agriculture Organization of the United Nations.

Gillett, R. \& Moy, W. (2006). Spearfishing in the Pacific Islands, Current Status and Management Issues. (FAO/FishCode Review. No. 19). Rome: Food and Agriculture Organization of the United Nations.

Graham, N. J., Wilson, S., Jennings, S., Polunin, N., Robinson, J., Bijoux, J., \& Daw, T. (2007). Lag effects in the impacts of mass coral bleaching on coral reef fish, fisheries, and ecosystems. Conservation Biology, 21, 1291-1300.

Hawkins, J. P. \& Roberts, C. (2004). Effects of Artisanal Fishing on Caribbean Coral Reefs. Conservation Biology, 18, 215-226.

Herrera-Ulloa, A., Villalobos-Chacón, L., Palacios-Villegas, J., Viquez-Portuguéz, R., \& Oro-Marcos, G. (2011). Coastal fisheries of Costa Rica. In S. Salas, R. Chuenpagdee, J. C. Seijo, \& A. Charles (Eds.), Coastal Fisheries of Latin America and the Caribbean (pp. 137-153). (FAO Fisheries and Aquaculture Technical Paper. No. 544). Rome: Food and Agriculture Organization of the United Nations.

Hobday, A. L., Tegner, M., \& Haaker, P. (2001). Overexploitation of a broadcast spawning invertebrate: decline of the white abalone. Reviews in Fish Biology and Fisheries, 10, 493-514.

Horta e Costa, B., Batista, M. I., Goncalves, L., Erzini, K., Caselle, J. E., Cabral, H. N., \& Goncalves, E. J. (2013). Fishers' Behaviour in Response to the Implementation of a Marine Protected Area. PLOS ONE, 8(6), e65057. doi:10.1371/journal.pone.0065057
Horta, S. \& Defeo, O. (2012). The spatial dynamics of the whitemouth croaker artisanal fishery in Uruguay and interdependencies with the industrial fleet. Fisheries Research, 125-126, 121-128.

Huchim-Lara, R., Fraga, J., \& Salas, S. (2012). Fishermen Social and Cultural Behavior Associated to Decompression Sickness in Lobster Fisheries from the Eastern Coast of Yucatan. Proceedings of the Gulf and Caribbean Fisheries Institute, 64, 142-146.

Instituto Nacional de Estadística y Censos. (2012). Estadísticas por región. San José, Costa Rica. Disponible en http://www.inec.go.cr/Web/Home/pagPrincipal.aspx

Karlson, R. H. \& Levitan, D. (1990). Recruitment-limitation in open populations of Diadema antillarum: an evaluation. Oecologia, 82, 40-44.

Katsanevakis, S., Maravelias, C. D., Vassilopoulou, V., \& Haralabous, J. (2010). Boat seines in Greece: landings profiles and identification of potential métiers. Scientia Marina, 74, 65-76.

Kronen, M. (2004). Fishing for fortunes?: A socio-economic assessment of Tonga's artisanal fisheries. Fisheries Research, 70, 121-134.

Lopes, P. F. M. \& Begossi, A. (2011). Decision-making processes by small-scale fishermen on the southeast coast of Brazil. Fisheries Management and Ecology, 18, 400-410.

Manly, B. F. (1991). Randomization and Monte Carlo Methods in Biology. New York, U.S.A.: Chapman \& Hall.

Maunder, M. N. \& Punt A. E. (2004). Standardizing catch and effort data: a review of recent approaches. Fisheries Research, 70, 141-159.

Moberg, F. \& Folke, C. (1999). Ecological goods and services of coral reef ecosystem. Ecological Economics, 29, 215-233.

Monroy, C., Salas, S., \& Bello-Pineda, J. (2010). Dynamics of fishing gear and spatial allocation of fishing effort in a multispecies fleet. North American Journal of Fisheries Management, 30, 1187-1202.

Morsan, E. M. (2007). Spatial pattern, harvesting and management of the artisanal fishery for purple clam (Amiantis purpurata) in Patagonia (Argentina). Ocean \& Coastal Management, 50, 481-497.

Moses, B. S., Udoidiong, O. M., \& Okon, A. (2002). A statistical survey of the artisanal fisheries of southeastern Nigeria and the influence of hydroclimatic factors on catch and resource productivity. Fisheries Research, 57, 267-278.

Naranjo, M. H. (2010). Caracterización de los sistemas operacionales, modalidades y artes de pesca utilizados para la captura de la langosta Panulirus gracilis (Streets, 1871) en Guanacaste, Costa Rica. Revista Ciencias Marinas y Costeras, 2, 73-82. 
Naranjo, M. H. (2011). Biología pesquera de la langosta Panulirus gracilis (Streets, 1871) en Playa Lagarto, Guanacaste, Costa Rica. Revista de Biología Tropical, 59, 619-633.

Naranjo, M. H. (2012). Patrón reproductivo y talla media de madurez sexual de hembras de la langosta Panulirus gracilis (Decapoda: Palinuridae) en Playa Lagarto, Guanacaste, Costa Rica. Revista de Biología Tropical, 60, 1783-1793.

Navarte, M., González, R., \& Filipo, P. (2007). Artisanal mollusk fisheries in San Matías Gulf (Patagonia, Argentina): An appraisal of the factors contributing to unsustainability. Fisheries Research, 87, 68-76.

Plagányi, E., van Putten, I., Hutton, T., Deng, R. A., Dennis, D., Pascoe, S., Skewes, T., \& Campbell, R. A. (2013). Integrating indigenous livelihood and lifestyle objectives in managing a natural resource. PNAS, 110, 3639-3644.

Pollnac, R. B. \& Poggie, J. (2008). Happiness, well-being and psychocultural adaptation to the stresses associated with marine fishing. Human Ecology Review, 15, 194-200.

Purcell, S., Mercier, A., Conand, C., Hamel, J., \& ToralGranda, M. (2011). Sea cucumber fisheries: global analysis of stocks, management measures and drivers of overfishing. Fish and Fisheries, 14, 34-59.

R Core Team. (2012). R: A language and environment for statistical computing. R Foundation for Statistical Computing, Vienna, Austria. v.2.15.0. ISBN 3-900051-07-0, URL http://www.R-project.org/

Riolo, F. (2006). A geographic information system for fisheries management in American Samoa. Environmental Modelleling \& Software, 21, 1025-1041.

Ríos-Lara, V., Salas, S., Javier, B., \& Ayora, P. (2007). Distribution patterns of spiny lobster (Panulirus argus) at Alacranes reef, Yucatan: Spatial analysis and inference of preferential habitat. Fisheries Research, $87,35-45$.

Sabetian, A. (2010). Parrotfish Fisheries and Population Dynamics: A Case Study from Solomon Islands. (Doctoral Dissertation). James Cook University, Australia. Retrieved from http://researchonline.jcu.edu. $\mathrm{au} / 11744 /$

Salas, S. \& Gaertner, D. (2004). The behavioural dynamics of fishers: Management implications. Fish and Fisheries, 5, 153-167.

Salas, S., Chuenpagdee, R., Seijo, J. C., \& Charles, A. (2007). Challenges in the assessment and management of small-scale fisheries in Latin America and the Caribbean. Fisheries Research, 87, 5-16.
Salas, S., Chuenpagdee, R., Seijo, J. C., \& Charles, A. (2011). Coastal Fisheries of Latin America and the Caribbean. (FAO Fisheries and Aquaculture Technical Paper. No. 544). Rome: Food and Agriculture Organization of the United Nations.

Sadovy, Y. (2005). Trouble on the reef: the imperative for managing vulnerable and valuable fisheries. Fish and Fisheries, 6, 167-185.

Shester, G. G. (2010). Explaining catch variation among Baja California lobster fishers through spatial analysis of trap-placement decisions. Bulletin of Marine Science, 86, 479-498.

Smith, S., Jacob, S., \& Jepson, M. (2003). After the Florida net ban: the impacts on commercial fishing families. Society \& Natural Resources, 16, 39-59.

Stevenson, T. C., Brian, N., \& Dierking, J. (2011). Fisher behaviour influences catch productivity and selectivity in West Hawaii's aquarium fishery. ICES Journal of Marine Science, 68, 813-822.

van Putten, I. E., Kulmala, S., Thébaud, O., Dowling, N., Hamon, K. G., Hutton, T., \& Pascoe, S. (2012). Theories and behavioural drivers underlying fleet dynamics models. Fish and Fisheries, 13, 216-235.

Voges, E., Gordoa, A., \& Field, J. (2005). Dynamics of the Namibian hake fleet and management connotations: applications of the ideal free distribution. Scientia Marina, 69, 285-293.

Wang, Y., Neuman, U., Wright, S. T., \& Warton, D. I. (2012). mvabund: an $\mathrm{R}$ package for model-based analysis of multivariate abundance data. Methods in Ecology and Evolution, 3, 471-474.

Warton, D. I., Wright, S. T., \& Wang, Y. (2012). Distancebased multivariate analyses confound location and dispersion effects. Methods in Ecology and Evolution, 3, 89-101.

Whitley, D. (1994). A genetic algorithm tutorial. Statistics and Computing, 4, 65-85.

Wood, S. N. (2006). Generalized Additive Models: An Introduction with $R$. New York, U.S.A.: Chapman \& Hall/CRC.

Worm, B., Hilborn, R., Baum, J. K., Branch, T. A., Collie, J. S., Costello, C., Fogarty, M. J., Fulton, E. A., Hutchings, J. A., Jennings, S., Jensen, O. P., Lotze, H. K., Mace, P. M., McClanahan, T. R., Minto, C., Palumbi, S. R., Parma, A. M., Ricard, D., Rosenberg, A. A., Watson, R., \& Zeller, D. (2009). Rebuilding global fisheries. Science, 325, 578-585.

Zuur, F. A., Leno, E., Walker, N. J., Saveliev, A., \& Smith, G. M. (2009). Mixed effects models and extensions in ecology with R. New York, U.S.A.: Springer Science. 